MATEC Web of Conferences 31, 08002 (2015)

DOI: $10.1051 /$ matecconf/ 20153108002

(C) Owned by the authors, published by EDP Sciences, 2015

\title{
Design of Wireless Remote Control Solar Cleaner
}

\author{
Ma Zhicai , Li Ling , Fu yanliang ,Tao Shaoshu, Cui Zhenyue, Bi Lulu \\ College of Electrical and Information Engineering, Liaoning Institute of Science and Technology, Benxi, Liaoning
}

\begin{abstract}
This paper describes the operation principle and the hardware composition of the wireless remote control cleaner by adopting solar panel power supply mode in detail. The experimental circuit is designed and on based on the designed experimental circuit the wireless remote control solar cleaner is made. The experimental results show that it is feasible that the combination of solar panels and wireless remote control cleaner.
\end{abstract}

\section{Introduction}

With the rapidly development of urbanization, high-rise buildings are becoming more and more. How to efficiently, safely clean work of high-rise building has gradually taken by the people. At present domestic and foreign majority remain in the manual cleaning that low efficiency and poor security. The development of robot technology makes the cleaning work possible automation of high-rise building. For example, foreign companies come up with clean with a mop for remote control. But a kind of climbing cleaner is developed at Harbin Institute of Technology for high-rise building glass curtain wall or tile wall. At present, the power of domestic and foreign remote cleaner part still adopts the rectification power supply or battery, but green renewable clean energy use less such as solar, namely photovoltaic technology. This paper is the Combination of wireless remote control car models and solar photovoltaic technology that realize the operation of wireless remote control solar cleaner by using solar power supply. The scheme is feasible that is verified by an experimental prototype. hardware structure and work mechanism of cleaner.

\section{Hardware Structure of Cleaner}

Wireless remote control solar cleaner is designed in this paper is mainly composed of a transmitter and a receiver and a motor and its drive circuit and power supply. The transmitter mainly comprises a coding circuit and a transmitting circuit. The coding circuit is controlled by the operator or control switch that makes the coding circuit generates need by the manipulator with different frequency signal representative of the different control commands. The transmitter function is sent the control signal to the receiver that control the cleaner upper or lower and left or right to move .The receivers main processes the control signal from the transmitter and the received control signal will be amplified and shaped and

a Corresponding author: author@e-mail.org decoded, converted to the signal that the executive circuit can identify. And the power supply is provided at part two part, when the sun light is enough, the photovoltaic solar panels. While in cloudy or when the sun is not enough, a storage battery is the use of providing electrical energy to the cleaner.

\section{Work Principle of the Cleaner}

Wireless remote control transmitting circuit is designed in this paper mainly in the integrated block PT8A9778 as the core and other components, its working principle diagram as shown in figure 1. 3, 9 feet of Integrated block IC1 is the power supply; its 1,14 feet is the control signal input end to left and right turn; 4, 5 feet is control signal input end for the forward and backward; 6 feet is the control signal input end to acceleration; $2,7,10,13$ feet is the free end, 8,9 feet is the code signals output terminal; 11, 12 feet connect with external oscillating resistance. Transistor Q1C945 and crystal X1 constitute the carrier oscillator, the oscillation frequency is $35 \mathrm{MHz}$. After the power supply is switched on, the function key operation in the remote, the integrated block PT8A9778 of the corresponding control pins, internal functions corresponding gating, start coding code generated signal consistent with the operation of the function keys, and then by the pin 8 output control code pulse signal, while the 9 foot outputs high level control signal. emitting diode LED light, B pole of triode Q1C945 has a very high level signals and conducting work, carrier oscillator composed of triode Q1C945 and the crystal oscillator X1 works. The oscillation generated carrier frequency of $35 \mathrm{MHz}$, the capacitor $\mathrm{C} 3$ coupled to the B pole of the triode Q2C945. When the integrated block PT8A9778 10 feet output high level control code pulse signal, the triode Q2C945 is conducted, the function control code pulse signal and the $35 \mathrm{MHz}$ carrier signal generated by a crystal oscillator X1 and triode Q1C945 triode Q2C945 modulated and amplified by $\mathrm{C} 7$, an inductance L4, 
capacitance coupling launch out. Figure1 9V power supply will be replaced from the $9 \mathrm{~V}$ solar cell board.

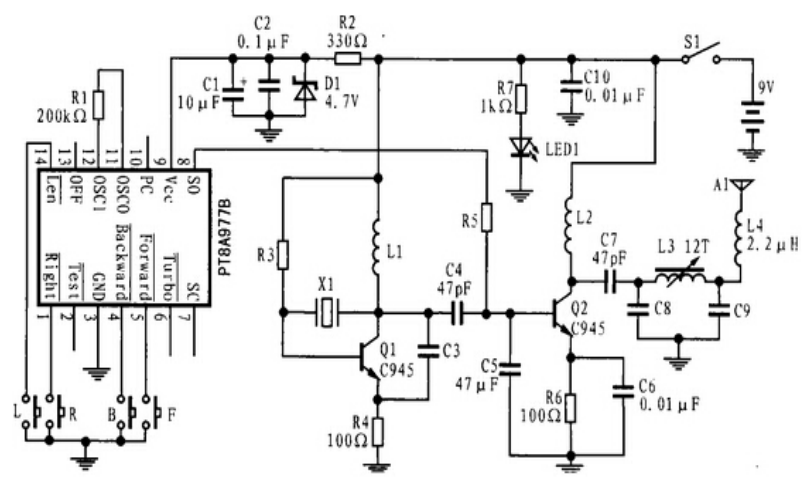

Fig. 1 Schematic diagram of wireless remote control transmitting circuit

Receiving circuit will handle wireless signals from the remote control transmitter and the signal will be amplied and shaped and coded, in the meantime the electrical signal changes into that executive circuit can be identified. The digital pulse signal transfers to other electronic components the trolley that the drives the motor to make car the upper and lower or left and right movement.

\section{Wireless Remote Control Solar Cleaner Prototype Experiment}

Based on the above principle, the design and fabrication of a wireless remote control solar cleaner prototype is shown in figure 2 . The prototype consists of three main parts respectively: the first part is solar panel and the other are remote controller and cleaner. The solar battery boards have two blocks of solar battery panels, a block of solar panel provides $+5 \mathrm{~V}$ power supply for cleaner power supply, and another block of solar panel provides $+9 \mathrm{~V}$ power supply to supply remote controller. Due to the device may work on a cloudy day or where light is much sufficient, so in the remote control and cleaner are equipped with rechargeable batteries, solar panels directly connected with a rechargeable battery.

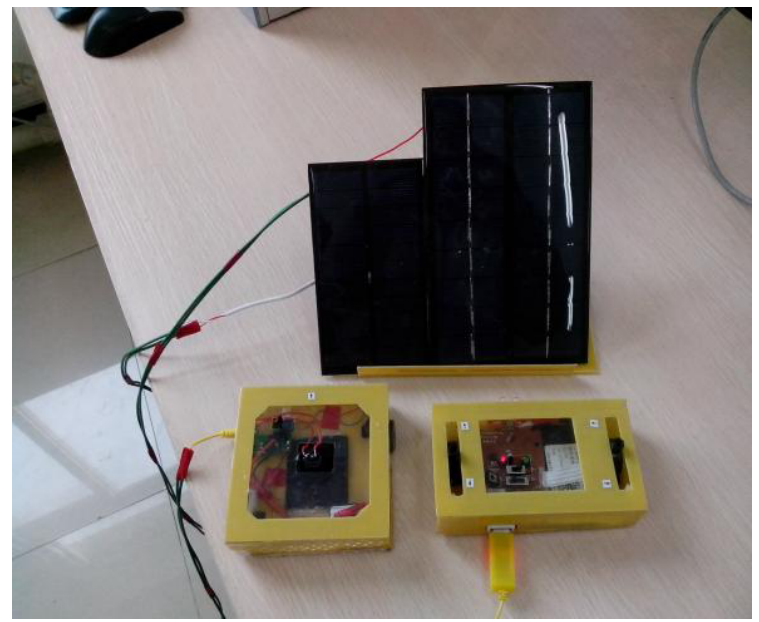

Fig. 2 wireless remote control solar cleaner prototype

According to the circuit diagram as shown in Figure 1 , the PCB circuit board of remote controller is made, and then welding the corresponding components, it made the remote controller as shown in figure 2. has a knob on each side that can control the cleaner upper and lower or left and right movement. The remote controller is equipped with USB interface, through this interface solar panels provide working power supply for remote controller.

The cleaner is a core part of the whole device, it consists of several parts : the wireless signal receiving circuit, driving circuit, motor and a centrifugal fan. Remote control car can climb the smooth wall mainly relying on the vacuum negative pressure principle and relying on centrifugal fan in the body that composed of a motor of the high-speed driving fan impeller rotation so that the air speed is discharged at the bottom, while the air at the bottom of the body is constantly added to the fan and cause the body internally generated instantaneous the vacuum, and the outside atmospheric pressure to form negative pressure difference. Under the pressure difference, climbing car firmly adsorbed on the walls, glass plane, so as to make the wall climbing car attached to the walls and other vertical or horizontal plane effect. And the power supply of cleaner other component is also provided by $+5 \mathrm{~V}$ solar panels. Back of cleaner stick the smooth cleaning dishcloth, On the one hand it can increase the sucker and the glass wall surface of the seal, on the other hand it can reduce the friction with the wall surface

When the cleaner is walking on glass, cleaning cloth can wipe off the dust on the glass. Figure 3 shows a wireless remote control solar cleaner prototype walking on the windows of the working drawing.

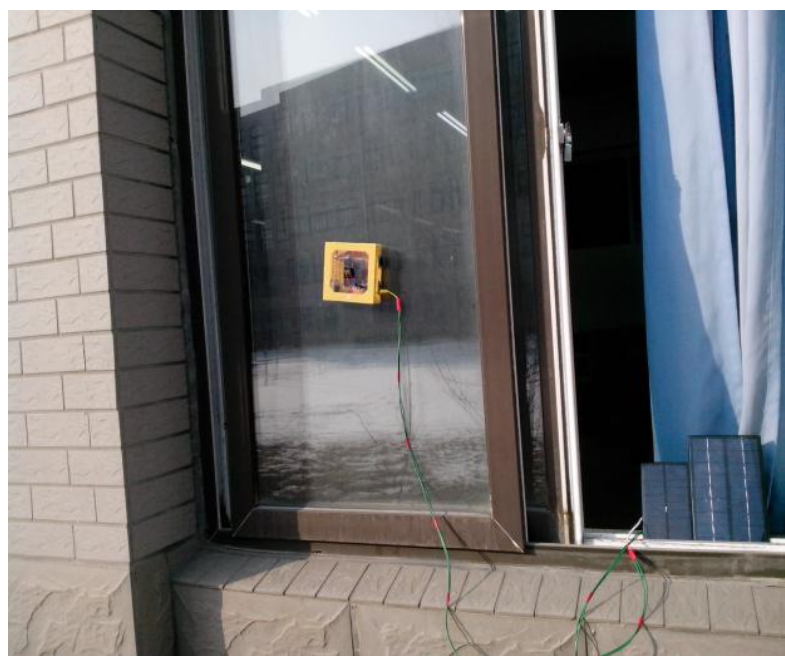

Fig. 3 wireless remote control solar cleaner prototype

\section{Conclusion}

The wireless remote-control cleaner that take the solar energy as energy is able to work in a vertical wall and ceiling of two states. Especially in the high-rise building glass curtain wall cleaning and cleaning 
work, play a greater role. Through theoretical analysis and experimental verification the design of the wireless remote control solar cleaner is reasonable and feasible, and has certain application value. Of course, the device also has some shortcomings and need to improve the place, such as with the development of solar energy technology solar panels and a cleaner can synthetizes one, which requires improvement in the design in the future.

\section{Acknowledgements}

This work was financially supported by Liaoning province college students' innovation and entrepreneurship training project (201411430009)

\section{References}

1. Xiao Li, Tong Shizhong, Ding Qimin etc. The present situation and development of wall climbing $\operatorname{robot}[\mathrm{J}]$. automation panorama, 2004 (1): 81-84.

2. Zhang Hua, Chen Maohua, Liu Nansheng etc. Development of crawler intelligent arc welding robot system[J], robot technique and application, 2002 (1): 13-16.

3. Meng Xianchao, Wang Zuwen, steel etc. The development of a multi sucker wall climbing robot prototype [J] .machine design, 2003 (8): 30-33.

4. Cai Zixing. The robotics [M]. Beijing: Tsinghua University press, 2000.

5. Huang Wenpan,Dai Yongxiong, Fan Maofei,Qiao Bin,Liu. Mingqin Research and production of wall climbing robot, Journal of Huaihai Institute of Techology (Natural Science Edition), 2010(2).

6. Pan Lei,Zhao Yanzheng,Qian Zhiyuan, Fu Zhuang,Cao Qixin.wall climbing robot adsorptioncharacteristics of has dual suction cups [J],Journal of Shanghai Jiao Tong University,2005(06).

7. $\mathrm{Wu}$ Shenli.Newbuilding cleaning research and design of the wall climbing robot[D].Chengdu University of Technology,2009

8. Gao Weiwei,Chen Zailiang etc.The principle of pneumatic transmission fivefoot climbing robot design and manufacture based on [J].Journal of Soochow University(Engineering Science Edition), 2006 (04).

9. Chen Peifu. Study on the design of glass curtain wall cleaning robot: Master thesis, Chongqing University, 2006. (5)

10.Zhang Zhaojun, Zhou Tingwu, Zong Guanghua. Application of window cleaning robot in the cleaningof high-rise building construction technology. 2001(9)

11. Wang Yuanyu, Take Rio, Li Yuanzong. Analysis of the development trend of wall cleaning robot. The introduction and consulting.2002(4).

12. Sao hao, Zhao Yan Zheng, Wang Yan, Liu shuxia, Liu Shuliang. Wall climbing robot system for glass curtain wall cleaning. Manufactu ring automation.2000 (2). 